\title{
Decoding the kinetic limitations of plasmon catalysis: the case of 4-nitrothiophenol dimerization
}

Wouter Koopman $^{a *}$, Radwan M. Sarhan ${ }^{a, b}$, Felix Stete $^{a, b}$, Clemens Schmitt $^{c}$, Matias Bargheer ${ }^{a, d}$

E-mail: wouter.koopman@uni-potsdam.de

Keywords: plasmon catalysis, photochemistry, DMAB, nitrothiophenol, reaction kinetics, reaction temperature, tandem reaction

\begin{abstract}
Plasmon-mediated chemistry presents an intriguing new approach to photocatalysis. However, the reaction enhancement mechanism is not well understood. In particular, the relative importance of plasmon-generated hot charges and photoheating are strongly debated. In this article, we evaluate the influence of microscopic photoheating on the kinetics of a model plasmon-catalyzed reaction: the light-induced 4-nitrothiophenol (4NTP) to 4,4'dimercaptoazobenzene (DMAB) dimerization. Direct measurement of the reaction temperature by nanoparticle Raman-thermometry demonstrated that the thermal effect plays a dominant role in the kinetic limitations of this multistep reaction. On the same time, no reaction is possible by dark heating to the same temperature. This shows that plasmon nanoparticles have the unique ability to enhance several steps of complex tandem reactions simultaneously. These results provide insight into the role of hot electron and thermal effects in plasmonic catalysis of complex organic reactions, which highly important for the ongoing development of plasmon based photosynthesis
\end{abstract}

a. Institute of Physics and Astronomy, University of Potsdam, Karl-Liebknecht-Str. 24-25, 14476 Potsdam, Germany

b. School of Analytical Sciences Adlershof (SALSA), Humboldt-Universität zu Berlin, Albert-Einstein-Str. 5-9, 10099, Berlin, Germany

c. Department of Biomaterials, Max Planck Institute of Colloids and Interfaces, Am Mühlenberg 1, 14476, Potsdam, Germany

d. Joint Research Group Ultrafast Dynamics, Helmholtz Zentrum Berlin, Albert-Einstein-Str. 15, 12489, Berlin, Germany 


\section{Introduction}

The purpose of a catalyst is to increase the rate of a reaction. A photo-catalyst does so by providing photo-excited electrons, effectively lowering the activation-energy. ${ }^{[1,2]}$ Additionally, excitation by light inevitably leads to photoheating, which can accelerate the reaction even further. ${ }^{[3,4]}$ The photocatalytic activity is therefore, at least in principle, controlled by increasing the light-intensity. In traditional semiconductor photocatalysis, heating causes thermally activated excited-state decay and charge recombination, ${ }^{[5]}$ which impedes the catalytic activity and limits the attainable turnover frequency.

Plasmon-driven photocatalysis by metal-nanoparticles offers an alternative approach that overcomes some of the limitations of traditional semiconductor photocatalysis. ${ }^{[6,7]}$ Plasmons are a resonant collective excitation of the conduction band-electrons of a metal nanoparticle. ${ }^{[8]}$ They decay by the formation of an energetic electron-hole pair, which has about the energy of the plasmon itself. ${ }^{[9-11]}$ One, or both, of the energetic carriers might subsequently transfer to a molecule adsorbed on the surface of the plasmonic structure. Alternatively, they thermalize leading to efficient nanoscale heating. ${ }^{[4,10]}$

Today, literature knows a large amount of reactions that are either plasmonically accelerated or even enabled. ${ }^{[12]}$ Examples range from the dissociation of $\mathrm{H}_{2},{ }^{[11,13]}$ via the reduction $\mathrm{H}_{2} \mathrm{O}{ }^{[14]}$ and of $\mathrm{CO}_{2},{ }^{[15]}$ to complex organic N-N or C-C coupling reactions. ${ }^{[16-25]}$ The latter reaction type is particularly interesting as it forms the basis for true photosynthetic capabilities.

Many of these studies have empirically shown a positive dependence of the plasmon-induced reaction rate on the light intensity ${ }^{[1,6]}$ and on the reaction temperature. ${ }^{[1,6,17]}$ Notably, the reason for the intensity dependence of the catalytic activity is strongly debated. ${ }^{[17,21,22,26,27]}$ The central controversy in this debate is the role of photoheating. While some authors systematically rule out any influence of photo-generated heat, ${ }^{[22]}$ others are convinced that they can explain the rate enhancement in plasmon-driven chemistry entirely as photoheating effect. ${ }^{[26,28]}$ In our opinion, 
this discussion frequently mixes up the question regarding the mechanism underlying the intensity dependence with the more general question if plasmon catalysis necessarily involves the generation of energetic (or "hot") electrons. We argue that on the contrary, these two questions must be examined separately.

All but the simplest examples of plasmon-driven reactions are multistep processes. In the dark, the rate limiting step of the reaction is obviously the activation. However, as the transfer of energetic electrons lowers this initial activation barrier, the reaction can be limited by a later step. A typical limitation in heterogeneous catalysis is for example the surface diffusivity of the activated reactants. ${ }^{[29]}$ Moreover, the reaction could be limited by the excitation of specific vibrations necessary to assume a molecular arrangement that allows the reaction to proceed. ${ }^{[18]}$ Increasing the generation of energetic electrons is of little use in this case.

In order to advance the discussion on the role of photoheating in plasmon-driven chemistry, we investigated the light-intensity dependence of the reaction rate of a deliberately complex multistep reaction: the photodimerization of 4-nitrothiophenol (4NTP) to 4-, 4'mercaptoazobenzne (DMAB) in the presence of gold nanoparticles. As this reaction has become somewhat the model instance for plasmon induced coupling reactions, ${ }^{[17-22,24,25,30]}$ it is of no surprise that several studies have already approached the influence photoheating in the past. Their results are however largely inconclusive..$^{[17,20-22]}$ On the one hand, Golubev et al. demonstrated that under reaction conditions that do not cause a reduction of 4NTP upon illumination, the reduction could be started by additionally increasing the temperature. ${ }^{[21]}$ Moreover, Zhang et al. presented a superlinear increase of the reaction rate on temperature, ${ }^{[20]}$ which can be interpreted as sign of a photothermal effect. On the other hand, seemingly opposing results were published by Keller and Frontiera, who measured the photoheating by ultrafast Raman thermometry. ${ }^{[22]}$ They did not observe a significant photoheating for longer than a few picoseconds after illumination. 
In this article, we discuss the correlation between the dependence of the reaction-rate on the light intensity and on an externally applied reaction temperature. Notably, we show that both dependencies are identical if the experimentally determined photoheating is considered, while at the same time, the dimerization cannot be achieved by pure external heating. These results demonstrate a large advantage of plasmonic photocatalysis: plasmonic nanoparticles can influence different steps of a reaction by different mechanism simultaneously.

\section{Results and Discussion}

The prominence of the plasmon-driven 4NTP dimerization in literature is largely caused by its facile observation using surface-enhanced Raman (micro-) spectroscopy (SERS): both the reactant and the product molecules have distinct Raman signatures. ${ }^{[17,31]}$

We measured the dimerization of 4NTP molecules chemisorbed on Au-Nanoflowers (Fehler! Ungültiger Eigenverweis auf Textmarke., inset) via an Au-S bond. Flowers were chosen for their irregular morphology, which enhances the SERS effect. ${ }^{[32]}$ To obtain comparable measurements, we used an area with a homogeneous distribution of particles as well as a large focal spot with a low magnification 10x objective (NA 0.25). Three main peaks dominate the typical Raman spectrum of 4NTP chemisorbed to Au nanoparticles (Fehler! Ungültiger Eigenverweis auf Textmarke.). The resonances at 1332, 1575, and $1097 \mathrm{~cm}^{-1}$ (R1-R3) correspond to the $\mathrm{NO}$ symmetric stretching, the ring $\mathrm{C}=\mathrm{C}$ stretching and the $\mathrm{C}-\mathrm{H}$ bending modes, respectively. ${ }^{[33]}$ Upon illuminating the particles with a $785 \mathrm{~nm} \mathrm{cw}-$ laser with an intensity of 159 $\mathrm{kW} / \mathrm{cm}^{2}$, additional Raman peaks at $1134 \mathrm{~cm}^{-1}, 1388 \mathrm{~cm}^{-1}$, and $1434 \mathrm{~cm}^{-1}$ (P1-P3) appear (Fehler! Ungültiger Eigenverweis auf Textmarke.). These can be assigned to vibrations involving the $\mathrm{N}=\mathrm{N}$ bond of DMAB that was formed by photo-dimerization of $4 \mathrm{NTP} .{ }^{[34]}$ Timeresolved Raman measurements (Fehler! Ungültiger Eigenverweis auf Textmarke.) show the kinetics of the DMAB-formation by the appearance of its characteristic resonances (P1-P3). 
Simultaneously, the intensity of the 4NTP fingerprint resonances (R1-R3) decreases. The DMAB fingerprint only occurs for a sufficiently strong illumination. Notably, it does not occur for an excitation with a light intensity below $4 \mathrm{~kW} / \mathrm{cm}^{2}$, even after a prolonged illumination period of $1 \mathrm{~h} \cdot{ }^{[17]}$ Neither does it occur by heating of the sample under dark conditions (see also Figure S1). Hence, the necessity of light for this reaction is obvious. As there is no qualitative difference between photoheating and external heating, photoheating alone cannot be the cause of the dimerization reaction. We hence assume that the reaction is started by transfer of energetic (non-thermal) charges that reduce the 4NTP.

Indeed, the dimerization of 4NTP most likely follows a redox scheme ${ }^{[24,25,35]}$ (Fehler! Verweisquelle konnte nicht gefunden werden.), which has been known since the 1980 from electrochemical investigations. ${ }^{[35]}$ In the plasmon-driven version of this reaction, the excitation of the particles provides the energetic electrons that are equivalent to the electrochemical charges. ${ }^{[25]}$ These reduce the 4NTP molecule, which eventually causes a hydrogen assisted cleavage of the oxygen atoms in the nitro-group. ${ }^{[35]}$

The over-all reaction proceeds via the formation of four different intermediates and the addition of each eight electrons and protons (Fehler! Verweisquelle konnte nicht gefunden werden.). As discussed earlier, we need to determine the rate limiting step from this reaction scheme to correctly interpret the kinetics. Having in mind the threshold-like dependence of the reaction on the light intensity, one might be tempted to assume that it is transfer of energetic electrons that limits the reaction rate. Consequently, many publications use quasi-single molecular kinetics to extract a reaction rate of dimerization. ${ }^{[20,23,25,36]}$ However, to be the limiting step, the activation by energetic electrons must be the slowest process in the reaction. This is rather unlikely, as the thermalization time of energetic electrons, which is the timescale in which the activation must occur, is in the order of a few fs. ${ }^{[8]}$ Even if more than one energetic electron would be required for the activation, this would not limit the reaction, as the duration between 
the absorption of two photons at the observed threshold intensity of $25 \mathrm{~kW} / \mathrm{cm}^{2}$ is about $0.5 \mathrm{ps}$ (see supp. info).

On the other hand, the formation of DMAB includes the bi-molecular dimerization step. Already the fact that the dimerization requires the presence of two different intermediates (DHTP and HATP), makes its occurrence less probable, and thus slower, than the prior single molecular steps. Moreover, Sun and coworkers recently showed that the dimerization requires a favorable geometrical arrangement of the reactants, ${ }^{[18]}$ which limits the rate even further.

For these reasons, the reaction is most likely limited by the dimerization step. Consequently, its kinetics should be described by a bi-molecular model. ${ }^{[24,37]}$ This interpretation is supported by a detailed statistical comparison, by van Schrojenstein-Lantman and coworkers. ${ }^{[24]}$ They compared the likeliness of a bi-molecular model and a mono-molecular model for the description of the DMAB formation kinetics and found that the bi-molecular description is clearly favorable.

We investigated the kinetics using the transients of the $P_{1}$ (a combined $\mathrm{N}=\mathrm{N}$ stretching and $\mathrm{C}$ $\mathrm{H}$ bending) and $R_{2}\left(\mathrm{NO}_{2}\right.$ symmetric stretching) resonances (

The prominence of the plasmon-driven 4NTP dimerization in literature is largely caused by its facile observation using surface-enhanced Raman (micro-) spectroscopy (SERS): both the reactant and the product molecules have distinct Raman signatures. ${ }^{[17,31]}$

We measured the dimerization of 4NTP molecules chemisorbed on Au-Nanoflowers (Fehler! Ungültiger Eigenverweis auf Textmarke., inset) via an Au-S bond. Flowers were chosen for their irregular morphology, which enhances the SERS effect. ${ }^{[32]}$ To obtain comparable measurements, we used an area with a homogeneous distribution of particles as well as a large focal spot with a low magnification 10x objective (NA 0.25). Three main peaks dominate the typical Raman spectrum of 4NTP chemisorbed to Au nanoparticles (Fehler! Ungültiger Eigenverweis auf Textmarke.). The resonances at 1332, 1575, and $1097 \mathrm{~cm}^{-1}$ (R1-R3) 
correspond to the $\mathrm{NO}$ symmetric stretching, the ring $\mathrm{C}=\mathrm{C}$ stretching and the $\mathrm{C}-\mathrm{H}$ bending modes, respectively. ${ }^{[33]}$ Upon illuminating the particles with a $785 \mathrm{~nm} \mathrm{cw}-$ laser with an intensity of 159 $\mathrm{kW} / \mathrm{cm}^{2}$, additional Raman peaks at $1134 \mathrm{~cm}^{-1}, 1388 \mathrm{~cm}^{-1}$, and $1434 \mathrm{~cm}^{-1}$ (P1-P3) appear (Fehler! Ungültiger Eigenverweis auf Textmarke.). These can be assigned to vibrations involving the $\mathrm{N}=\mathrm{N}$ bond of DMAB that was formed by photo-dimerization of $4 \mathrm{NTP} .{ }^{[34]}$ Timeresolved Raman measurements (Fehler! Ungültiger Eigenverweis auf Textmarke.) show the kinetics of the DMAB-formation by the appearance of its characteristic resonances (P1-P3). Simultaneously, the intensity of the 4NTP fingerprint resonances (R1-R3) decreases. The DMAB fingerprint only occurs for a sufficiently strong illumination. Notably, it does not occur for an excitation with a light intensity below $4 \mathrm{~kW} / \mathrm{cm}^{2}$, even after a prolonged illumination period of $1 h \cdot{ }^{[17]}$ Neither does it occur by heating of the sample under dark conditions (see also Figure S1). Hence, the necessity of light for this reaction is obvious. As there is no qualitative difference between photoheating and external heating, photoheating alone cannot be the cause of the dimerization reaction. We hence assume that the reaction is started by transfer of energetic (non-thermal) charges that reduce the 4NTP.

). These are least affected by crosstalk from other resonances and hence reflect the pure reactant and product kinetics most clearly. As 4 NTP does not have any resonance at $1134 \mathrm{~cm}^{-1}$, the $P_{1}$ peak at this wavenumber should exclusively show the generation of DMAB. Indeed, the experimental transients have only one timescale (Fehler! Verweisquelle konnte nicht gefunden werden., orange squares).

On the other hand, the slope of the $R_{2}$-transients suggest the presence of a fast and a slow timescale (Fehler! Verweisquelle konnte nicht gefunden werden., blue squares). As the second timescale is not present in the $P_{1}$-transients, we assume that next to the DMAB formation a second reaction pathway for 4NTP exists. We found some evidence in literature that the 4NTP reduction and the DMAB formation only occur at plasmonic hotspots. ${ }^{[17,38,39]}$ 
Conversely, this means that the largest fraction of 4NTP molecules does not react to DMAB but follows a different reaction pathway. Possible reactions are the direct formation of 4aminothiopehnol (4ATP) ${ }^{[25]}$ or the plasmon assisted cleaving of the nitro-group to form thiophenol (TP). ${ }^{[39]}$ The latter has been demonstrated for Au nanoparticles with such a low 4NTP coverage that the reactants were too far separated to find a reaction partner.

We believe that DMAB can only be formed in a gap between two particles, as otherwise the trans-configuration of the molecules cannot be realized. At points that are not directly adjacent to another particle, the 4NTP molecules hence cannot form DMAB. Consequently, they react to another product. As the Raman cross-sections of both possible products (4ATP and TP) as well as the SERS enhancement outside of hotspots are low compared to the situation for DMAB, it is difficult to observe the appearance of the second product directly.

The amount of 4NTP and DMAB at a given time $t$, depends on the second order rate constant $k_{2}$ and the initial amount of the reactant $[R]_{0}$. As discussed earlier, not all the initial 4NTP molecules convert to DMAB, hence we describe $[R]_{0}$ by the sum of three fractions $[R]_{0, \text { dim }}$, $[R]_{0, \text { diss }}$ and $[R]_{0, \text { inert }}$ that represents the amount of 4NTP molecules that dimerize to DMAB, dissociate to another product with a rate $k_{1}$ or are completely inert, respectively. Multiplying the obtained reactant and product amounts with the Raman-cross-sections $\sigma_{R_{2}}$ and $\sigma_{P_{1}}$ then gives the Raman intensity for $R_{2}$ and $P_{1}$ peaks. The time-dependent Raman signal for the formation of DMAB thus follows the relation (see also supporting information for a detailed derivation):

$I_{P}(t)=\frac{1}{2} \cdot \sigma_{\mathrm{P}_{1}} \frac{[R]_{0, \mathrm{dim}}^{2} k_{2} t}{1+[R]_{0, \mathrm{dim}} k_{2} t}=\sigma_{P}^{\prime} \cdot \frac{K_{2} t}{1+K_{2} t}$

In the fitting procedure we used the adjusted rate constant $K_{2}=[R]_{0, c a t} k_{2}$, which has the dimension $1 / \mathrm{s}$. Moreover, we used the total Raman scattering $\sigma_{P}^{\prime}=\sigma_{P_{1}} \cdot[R]_{0, \operatorname{dim}} / 2$, which corresponds to the total Raman signal after all 4NTP molecules have been converted. 
The transient Raman signal corresponding to the 4NTP consumption is best described by the presence of two parallel reactions. The first order reaction describes the dissociation and the second order reaction the dimerization process. A global offset finally considers the inert fraction of 4NTP molecules that do not react at all:

$$
\begin{array}{rlc}
I_{R}(t) & = & \frac{\sigma_{R, \text { dim }}^{\prime}}{1+\mathrm{K}_{2} t} \\
& + & \sigma_{R, \text { diss }}^{\prime} \cdot e^{-k_{1} t} \\
& + & \sigma_{R, \text { inert }}^{\prime}
\end{array}
$$

The total Raman signal parameters $\sigma_{R, \text { dim }}^{\prime}, \sigma_{R, \text { diss }}^{\prime}$ and $\sigma_{R, \text { inert }}^{\prime}$ in equation 2 are defined corresponding to $\sigma_{P}^{\prime}$. The model given by equation 1 and equation 2 , indeed describes the $R_{2^{-}}$ and $P_{1}$-transients very well (Fehler! Verweisquelle konnte nicht gefunden werden.). Notably, a single value for $K_{2}$ was chosen for the DMAB production (equation 1) and the 4NTP consumption (equation 2). This description by a single rate for consumption and production is not possible using a mono-molecular model, which has led to some confusion in the past. ${ }^{[23]}$ We hence take the simultaneously good agreement of both resonances as a strong indicator for the correctness of our bi-molecular approach.

Having established an understanding of the reaction kinetics, we now turn our attention to the dependence of $K_{2}$ on the light intensity. In principle, two intensity-dependent mechanisms could influence $K_{2}$. For once, increasing the light intensity causes an increased number of energetic electrons. These can in turn activate a larger number of reactants that are subsequently able to undergo the dimerization step. On the other hand, illuminating plasmonic particles leads to a significant (local) temperature increase, ${ }^{[17]}$ which can increase the dimerization velocity. To understand the role of photoheating, we compared the dependence of $K_{2}$ on light intensity $I$ with its dependence on an external reaction temperature $T_{\text {ext }}$ (Figure 3 a,b) applied by a heating stage. It becomes immediately clear, that in both cases $K_{2}$ follows a super-linear 
dependence. Indeed, the similarity between the slopes of $K_{2}(I)$ and $K_{2}\left(T_{\text {ext }}\right)$ suggest that both follow a thermal Boltzmann distribution.

To directly compare $K_{2}(I)$ and $K_{2}\left(T_{\text {ext }}\right)$, it is essential to determine the amount of photoheating induced by illuminating the particles. Boerigter et al. demonstrated a direct measurement of the nanoparticle-temperature by Raman-thermometry. ${ }^{[40]}$ In short, photons scattered from the Fermi-Dirac distributed electrons lead to a Boltzmann-distributed background intensity in the anti-Stokes signal. Using this method, we recently demonstrated a significant increase in the nanoparticle temperature during the 4NTP dimerization. ${ }^{[17]}$ Here we go one step further - we used the photoheating measured by Raman thermometry to determine a heat conversion factor for calculating the nanoparticle temperature for a given light intensity. By a linear fitting of the measured particle-temperature for four intensities, we determined a conversion factor of $0,82 \mathrm{~K} \cdot \mathrm{cm}^{2} / \mathrm{kW}$ (details see supporting information).

The experimentally determined photoheating factor allows us to calculate the light-induced temperature increase $\Delta T_{\text {photo }}$ for each intensity. Figure 3c shows the dependence of $K_{2}$ on the photoheating $\left(K_{2}\left(\Delta T_{\text {photo }}\right)\right.$, red $)$ and on external heating $\left(K_{2}\left(T_{\text {ext }}+\Delta T_{\text {photo }}\right)\right.$, blue $)$ in the form of an Arrhenius plot. In the latter case, $\Delta T_{\text {photo }}$ amounts to the temperature increase caused by a Raman-laser of $5 \mathrm{~mW}$. The Arrhenius plot is a linearization of the exponential dependence of a reaction-rate $K$ on the reaction temperature $T$, according to the Arrhenius formula:

$$
K=A \cdot e^{-\frac{E_{A}}{k_{B} T}}
$$

Here $E_{A}$ is the activation energy of the reaction, $k_{B}$ the Boltzmann-constant and the preexponential factor $A$ the attempt frequency. A linear slope in this representation is thus a strong indicator for thermal origin of the rate-variation.

No statistics is necessary to immediately recognize that both measurements lie on one line and have the same activation energy. Indeed, a linear fitting gives for both measurements an 
activation energy of $305 \mathrm{meV}$. Moreover, all datapoints lie within the $95 \%$ prediction interval of both fittings. We therefore assume with good confidence, that both measurements show the same physical mechanism - which must be heating.

Immediately, the following question arises: if the dependence of $K_{2}$ on the light-intensity is exclusively explained by photoheating, how can it be understood that no reaction occurs in the absence of light - even at high temperatures? In our opinion the answer to this question can be found in the mechanisms limiting the reaction rate (Figure 4). The threshold-like behavior of the reaction with light intensity suggests that the reaction needs to be activated by energetic electrons. However, as the activation energy for the first step is lowered, a later reaction-step, the bi-molecular combination of DHBT and HABT, limits the reaction rate. In order to overcome the activation barrier, the molecules need to assume a beneficial conformation, which is probably different form their equilibrium conformation. ${ }^{[18]}$ Increasing the temperature provides more vibrational energy, so the molecules can rearrange into a geometrical conformation that fosters the formation of DMAB.

\section{Conclusions}

In conclusion we discuss the role of photoheating for the light-intensity dependence of a prototypical plasmon-driven coupling reaction - the photodimerization of 4NTP to DMAB. We established that the chemical kinetics of this reaction is limited by the bi-molecular combination of two activated 4NTP molecules. We experimentally determined a photoheating factor to calculate the temperature increase when increasing the light intensity. This allows a direct comparison between the influence of different light intensities and different reaction temperatures on the reaction rate. The comparison shows an Arrhenius-like behavior with identical activation energies in both cases. Thus, the rate of the full reaction is only determined by the particle heating and not by the amount of available energetic electrons. We explain this observation by the fact that the over-all reaction is limited by the bi-molecular dimerization step 
rather than the initial electron injection step. The dimerization step is likely limited by the geometrical conformation and possibly the surface diffusion of reactants and hence strongly temperature dependent.

The investigation at hand shows that plasmonic nanoparticles can function as "tandem catalysts", influencing reactions in different ways simultaneously. This means that great care must be taken to understand the mechanism and kinetics of a given plasmon induced reaction, in order understand the different influences the nanoparticle rector. It also demonstrates a great advantage of plasmon photocatalysis over traditional semiconductor approaches: plasmonic nanoparticles can simultaneously foster different reaction steps by different mechanism

\section{Experimental Section}

Synthesis of gold nanoflowers (AuNFs): We prepared irregularly shaped gold nanoparticles, known as AuNFs in literature, according to an established procedure. ${ }^{[17,32]}$ Briefly, we mixed $200 \mu 1$ of $100 \mathrm{mM}$ of HEPES buffer solution (pH 7.4+ 0.5) thoroughly with $1.8 \mathrm{~mL}$ water for $5 \mathrm{~min}$. Then, $40 \mu \mathrm{l}$ of $25 \mathrm{mM}$ aqueous solution of HAuCl4 was quickly added. The final mixture was left undisturbed for $2 \mathrm{~h}$. During this time, the color turned from pale yellow to colorless and finally to dark blue. The AuNFs were washed several times by centrifugation and finally dispersed in $500 \mu \mathrm{l}$ water.

Fabrication of SERS-substrate: $1 \mathrm{~cm}^{2}$ pieces were cut from silicon wafers and cleaned by immersion in a solution of $30 \mathrm{wt} \% \mathrm{H}_{2} \mathrm{O}_{2}$ and $30 \mathrm{wt} \% \mathrm{H}_{2} \mathrm{SO}_{4}$ for 1 hour and subsequent washing with ethanol and water. AuNFs were deposited by drop-casting $50 \mu 1$ of AuNF solution and subsequent drying for several hours. 4NTP molecules were self-assembled by immersion of the substrates in an ethanolic solution of $5 \mathrm{mM}$ of 4NTP for $6 \mathrm{~h}$. Finally, the substrates were washed with ethanol and water to remove the unattached molecules. 
SEM Microscopy: AuNFs were placed on a Si-substrate by drop-casting according to the procedure described in the last section. SEM micrographs were taken with the BSE detector of a Zeiss Gemini SEM at $1 \mathrm{keV}$.

Raman Micro-Spectroscopy: Raman spectra and kinetics were measured by a commercial confocal Raman microscope (alpha 300; WITec, Ulm, Germany), equipped with a thermoelectrically cooled CCD detector (DU401A-BV, Andor, UK) placed behind a spectrometer (UHTS 300; WITec, Ulm, Germany). The spectrometer was calibrated using the characteristic Raman band of the silicon wafer at $520 \mathrm{~cm}^{-1}$. A laser wavelength of $785 \mathrm{~nm}$ was chosen such that the excitation lies well separated from any direct vibronic excitation of the molecules, but still within the plasmon band of the particles. In order to minimize sample variations, we used a $10 \times($ Nikon, $\mathrm{NA}=0.25)$ microscope objective with a focal spot of roughly $2 \mu \mathrm{m}$. Temperature dependent Raman measurements were performed by placing the sample in a closed-chamber Linkam heating stage. All measurements showed a strong intensitydependent background, that might be connected to Au-NP fluorescence. ${ }^{[41]} \mathrm{We}$ used the PolyMod algorithm by Lieber and Madhadevan-Jansen ${ }^{[42]}$ with 50 iterations to fit the background by a $3^{\text {rd }}$ order polynomial. All subsequent analysis was done on backgroundcorrected spectra.

Raman Thermometry: Thermometry and kinetics measurement were, for technical reasons, not possible at the same setup. Raman thermometry was performed by simultaneously recording the anti-Stokes Raman spectra using a different confocal Raman microscope (JASCO NRS4100). Laser excitation wavelength and spot size were chosen identical to the kinetics measurements (785 $\mathrm{nm}$ and $2 \mu \mathrm{m}$ spot diameter). An intensity calibration delivered by the manufacturer was used to correct the wavelength-dependent sensitivity of the silicon CCD. Different excitation powers were achieved using neutral density filters. To determine the nanoparticle temperature a data-analysis procedure introduced by Boerigter et al. ${ }^{[0]}$ was 
employed. More information on this procedure can be found in the section "Measurement of Nanoparticle Temperature by Raman Thermometry" of the supporting information.

\section{Figures}

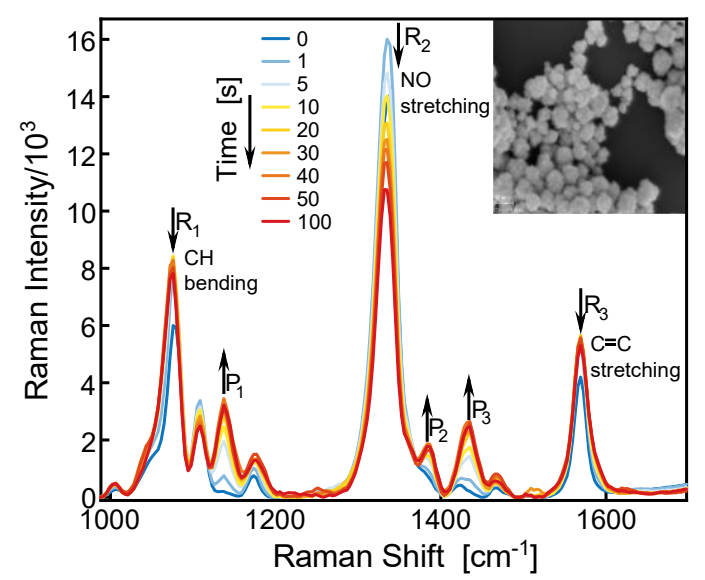

Figure 1. In the SERS spectrum the consumption of 4 NTP and the production of DMAB is observed by the decay and growth of their characteristic peaks R1, R2, R3 and P1, P2, P3, respectively. The inset shows a typical SEM micrograph of the Au nanoflowers. 
Scheme 1. The transformation of 4NTP to DMAB involves four intermediates and the addition of in total 8 electrons. The intermediates include dihydroxylaminothiophenol (DHATP), nitrosothiophenol (NSTP), hydroxylaminothiophenol (HATP) and azoxybenzenethiol $(\mathrm{AZOBT}) \cdot{ }^{[35]}$

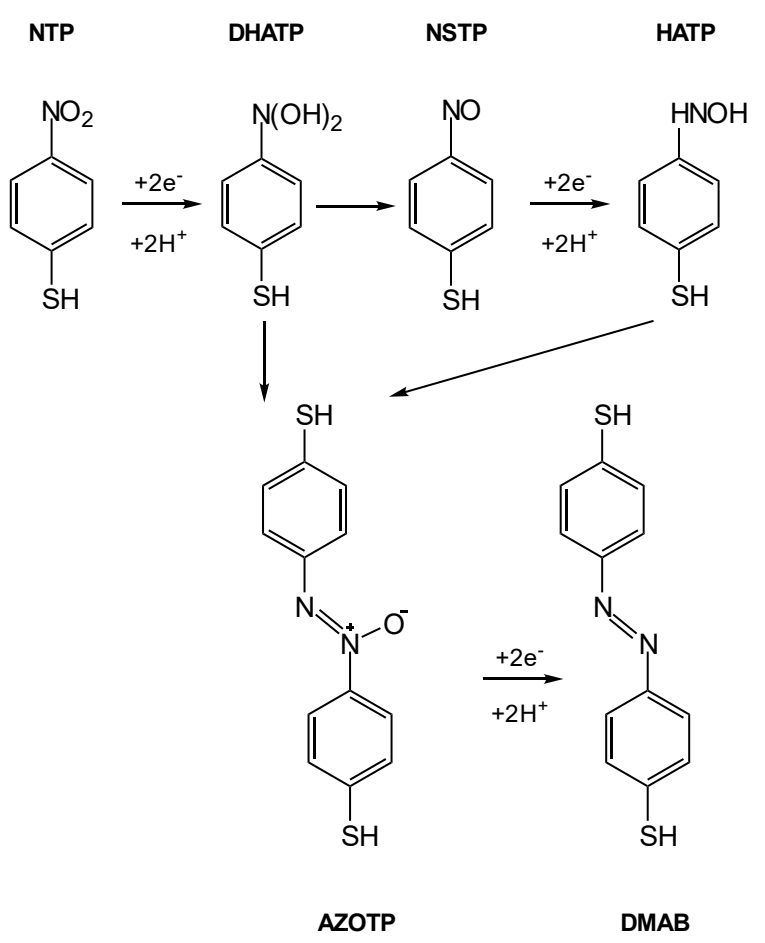




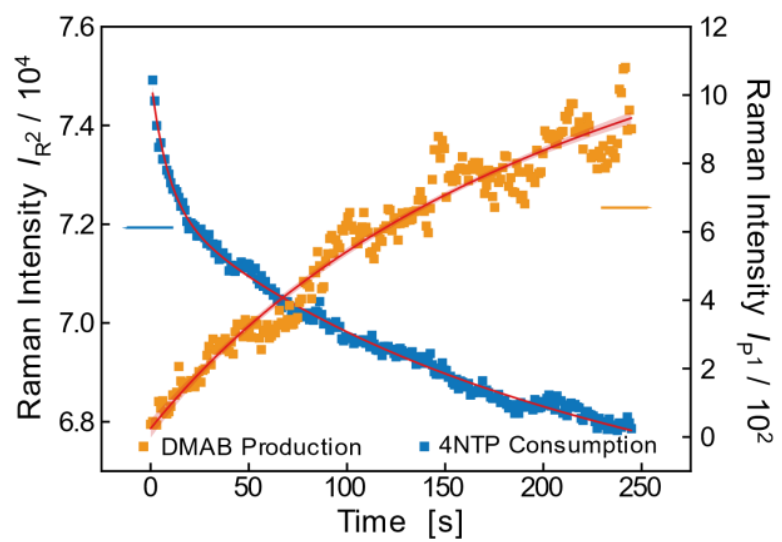

Figure 2. Transient of the DMAB production (orange) and 4NTP consumption (blue), obtained by integrating the R2 (blue) and P2 (orange) peaks.
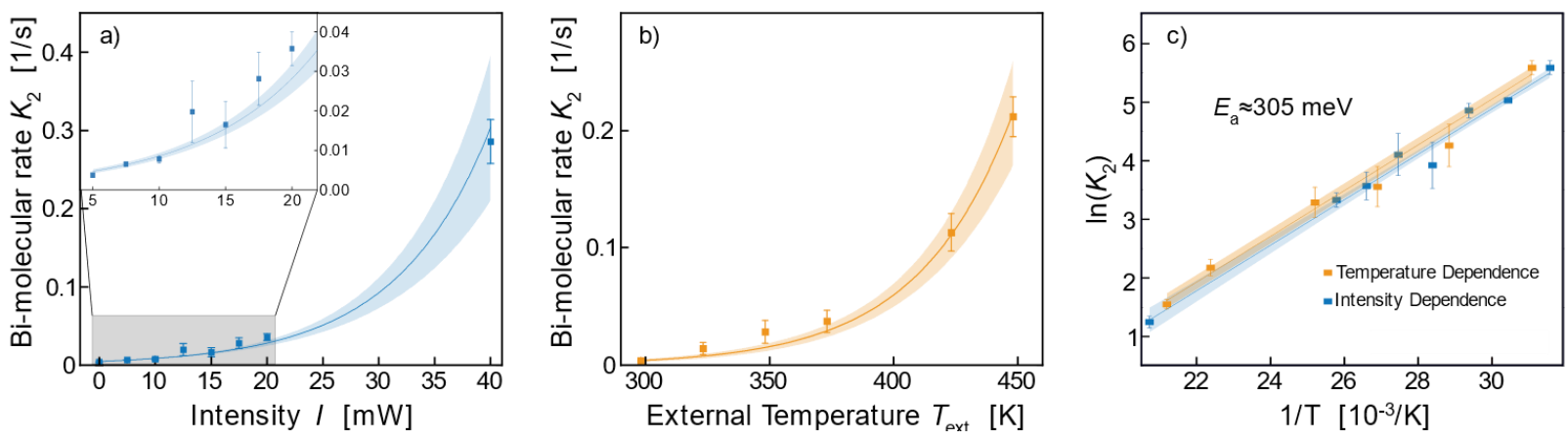

Figure 3. The bimolecular rate $K_{2}$ shows a superlinear dependence on both, the excitation intensity at room temperature (a) and on the reaction temperature by external heating at $5 \mathrm{~mW}$ excitation (b). In an Arrhenius plot (c), the reaction rates obtained by variation of intensity and temperature lie approximately on one line and have the same activation energy. The quasiexponential curves in (a) and (b) use the values obtained by the linear fitting in the Arrhenius plot. 


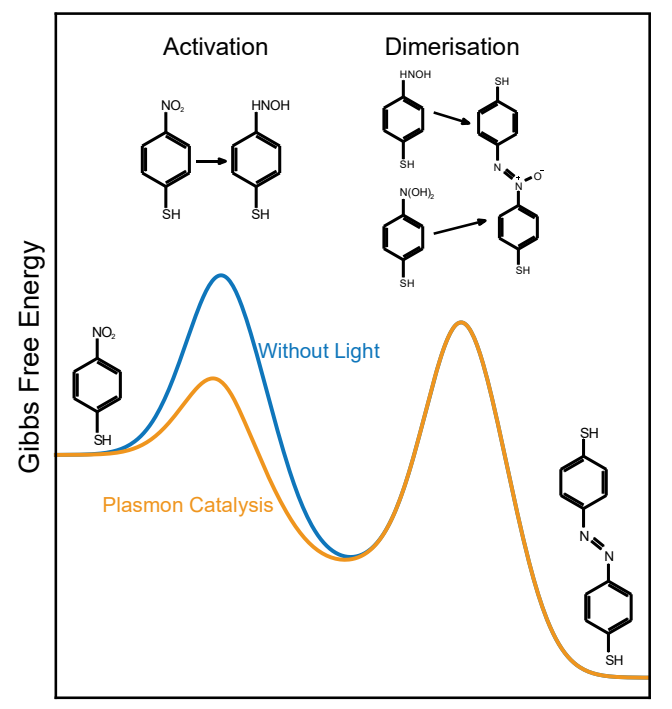

Reaction Progress

Figure 4. Schematics of the Gibbs free energy in a Plasmon-driven multistep reaction. As the plasmon excitation lowers the initial reaction barrier, the subsequent dimerization step limits the reaction rate. The activation energy determined in Figure 3c) is given by the dimerization barrier. 


\section{Supplementary Information}

\section{S1. Calculation of Absorption Time}

We assumed that the geometrical cross-section of the particles is about the same as their absorption cross-section ${ }^{[43]}$. In this case the photon arrival frequency $f_{\text {photon }}$ is:

$f_{\text {photons }}=I \cdot \pi r^{2} \cdot \frac{\lambda}{h c}=2 T H z$

with $I=25 \mathrm{~kW} / \mathrm{cm}^{2}, r=25 \mathrm{~nm}, \lambda_{\text {Laser }}=785 \mathrm{~nm}$. For the time between the arrival of two photons this gives $T_{\text {photon }}=1 / f_{\text {photons }}=0.5 \mathrm{ps}$, which is sufficient to trigger the reaction.

\section{S2. Low Intensity SERS}

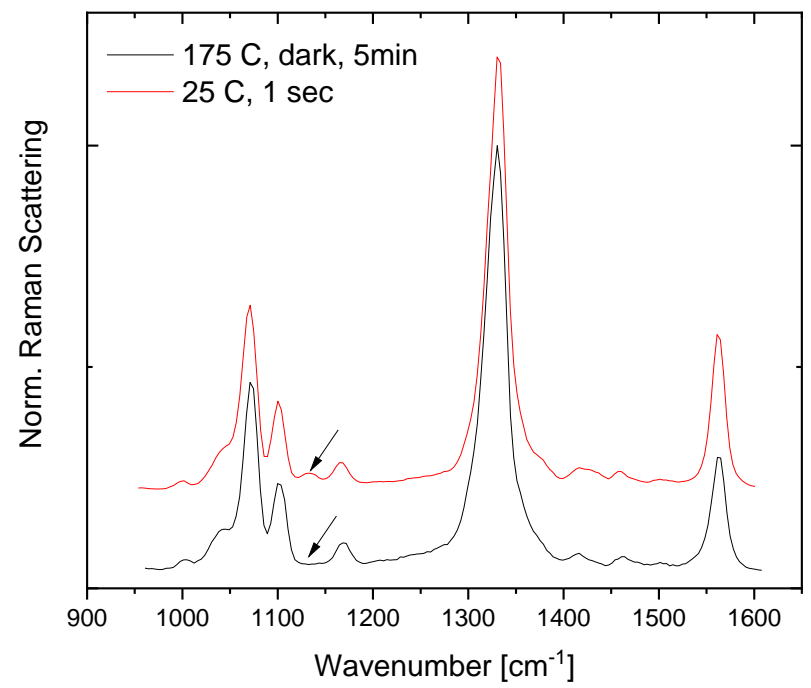

Figure S1. Comparison between Raman spectra taken after 5 min reaction time at $175^{\circ} \mathrm{C}$ under dark conditions (black line) and $1 \mathrm{sec}$ reaction time at $25^{\circ} \mathrm{C}$ with an illumination of $25 \mathrm{~kW} / \mathrm{cm}^{2}$.

\section{S3. Reaction Kinetics}

\section{S3.1 Product Peak $\boldsymbol{P}_{1}$}


The dimerization of two reactant molecules $R$ to one product molecule $P$, is a bimolecular reaction according to the scheme $\mathrm{e}^{[43]}$ :

$R+R \rightarrow P$

The kinetics of the reaction can be obtained from the corresponding differential equation:

$-2 \frac{d[P]}{d t}=\frac{d[R]}{d t}=-k_{2}[R]^{2}$

Were $[R]$ and $[P]$ are the concentrations of reactant and product and $k_{2}$ is the second order rate constant. This DGL must be integrated with respect to time to obtain an expression for reactant a given point in time $[R(t)]$ :

$\int_{R_{0}}^{R_{t}} \frac{d[R]}{[R]^{2}}=\int_{0}^{t}-k_{2} d t \Rightarrow-\left.\frac{1}{[R]}\right|_{R_{0}} ^{R_{t}}=-k t \Leftrightarrow \frac{1}{[R]_{0}}-\frac{1}{[R]_{t}}=k_{2} t$

This simplifies to:

$[R]_{t}=\frac{1}{k_{2} t+1 /[R]_{0}}=\frac{[R]_{0}}{1+[R]_{0} \cdot k_{2} t}$

The product concentration $[P(t)]$ at a given time $t$ is then obtained by:

$[P(t)]=\frac{[R]_{0}-\left[R_{t}\right]}{2}=\frac{[R]_{0}}{2}\left(1-\frac{1}{1+[R]_{0} k_{2} t}\right)=\frac{[R]_{0}}{2} \cdot \frac{\left([R]_{0} k_{2} t\right)}{1+[R]_{0} k_{2} t}=\frac{1}{2} \cdot \frac{[R]_{0}^{2} k_{2} t}{1+[R]_{0} k_{2} t}$

To obtain the Raman signal of the $P_{1}$ product peak of DMAB, we must multiply this concentration by the Raman cross-section of the peak $\sigma_{P_{1}}$. For simplicity, the cross-section shall also include the SERS enhancement factor. This approach is legitimate, as we expect that the enhancement does not change during the course of the reaction. For the Raman intensity of the peak $P_{1}$ we thus get:

$I_{P_{1}}(t)=\sigma_{P_{1}} \frac{1}{2} \cdot \frac{[R]_{0}^{2} k_{2} t}{1+[R]_{0} k_{2} t}+I_{0}$

Here $I_{0}$ is an additional fitting component, that takes into account a small non-zero background at the start of the reaction.

\subsection{Reactant Peak $\boldsymbol{R}_{2}$}


The fraction of the reactant peak $R_{2}$ that decays because of the production of DMAB can be easily obtained by multiplying equation $\mathrm{S} 5$ with the Raman cross-section of the $R_{2}$ peak $\sigma_{R_{2}}$. However, we assume that 4NTP does not only react to DMAB, but also has a parallel first order reaction route, which we must include to obtain an expression for the intensity $I_{R_{1}}(t)$. The corresponding differential equation is:

$\frac{d[R]}{d t}=-k_{1}[R]$

With the solution:

$[R]=[R]_{0} \cdot e^{-k_{1} t}$

The progression of the peak $R_{2}$ during the reaction is hence given by:

$$
\begin{array}{rcc}
I_{R}(t) & = & \sigma_{R_{2}} \frac{[R]_{0, \mathrm{dim}}}{1+[R]_{0, \mathrm{dim}} \cdot k_{2} t} \\
& +\sigma_{R_{2}}[R]_{0, \text { diss }} \cdot e^{-k_{1} t} \\
& + & \sigma_{R_{2}}[R]_{0, \text { inert }}
\end{array}
$$

Here $[R]_{0, \text { cat }}$ is the fraction of 4 NTP that dimerizes to DMAB, $[R]_{0, \text { diss }}$ is the fraction that follows the dissociative first order route and $[R]_{0, \text { inert }}$ is the fraction that does not react at all.

\section{S4. Measurement of Nanoparticle Temperature by Raman Thermometry}

We measured the electron temperature of the nanoparticle by the method promoted by Boerigter et al. ${ }^{[40]}$ In short, photons can scatter from the Fermi-Dirac distributed electrons in the nanoparticle. This gives a smooth inverse exponential background in the anti-Stokes Raman signal. Following their procedure, we fitted the anti-Stokes background by:

$I_{\text {aS, backgroud }}=I_{a S, 0}\left[e^{E_{\text {Raman }} / k_{B} T}+1\right]^{-1}$

To separate the background from the Raman signal of the adsorbate molecules, we used an approach inspired by the PolyMod algorithm by Lieber and Madhadevan-Jansen ${ }^{[42]}$. The basic idea of this algorithm is to fit a model-function to the data, and subsequently replace all data points whose value is larger than the fitted function value, by the function value. The thus 
obtained new dataset is then again fitted by the model-function and the replacement step is repeated. This procedure is repeated until a fitted function that only represents the background remains. In the original PolyMod algorithm, a polynomial is used as model function. For the temperature determination, we used instead the function described in equation S11.

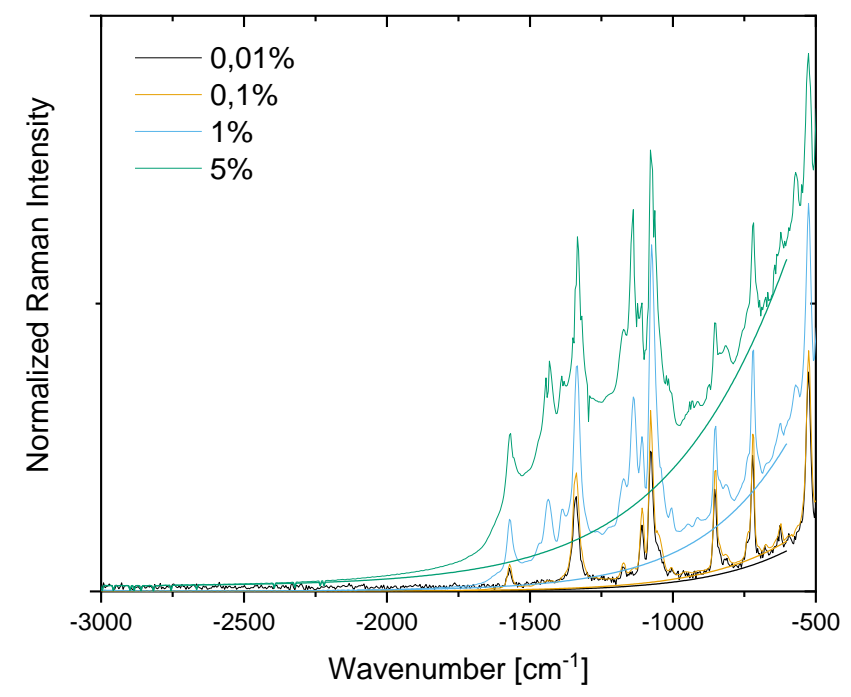

Figure S2. Anti-Stokes spectra at 4 different laser intensities and corresponding fits of the Fermi-Dirac background.

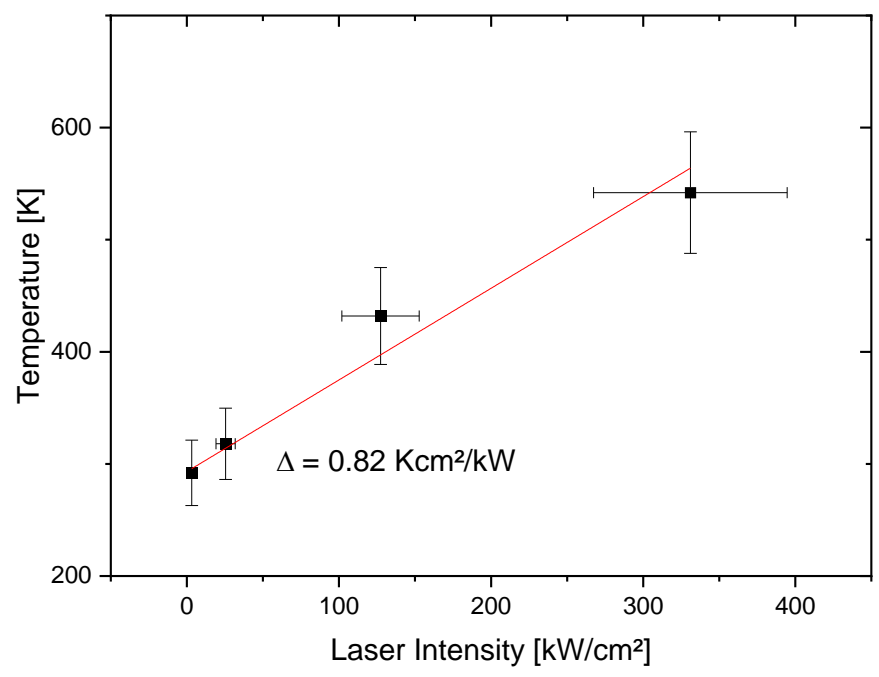


Figure S3. Particle temperatures extracted from anti-Stokes background versus the applied laser intensity. The linear fit has a fixed y-axis intercept at room temperature $\left(20^{\circ} \mathrm{C}\right)$.

\section{Acknowledgements}

R.M.S. and F.S. gratefully acknowledge the financial support by the DFG via the School of Analytical Science Adlershof (SALSA). We thank Ilko Bald, Peter Saalfrank and Bartholomäus Pieber for fruitful discussions.

\section{References}

[1] Y. Kim, D. Dumett Torres, P. K. Jain, Nano Lett. 2016, 16, 3399.

[2] S. Yu, P. K. Jain, Angewandte Chemie (International ed. in English) 2020, 59, 2085.

[3] a) M. Ghoussoub, M. Xia, P. N. Duchesne, D. Segal, G. Ozin, Energy Environ. Sci. 2019, 12, 1122; b) J. Qiu, W. D. Wei, J. Phys. Chem. C 2014, 118, 20735.

[4] J. R. Adleman, D. A. Boyd, D. G. Goodwin, D. Psaltis, Nano letters 2009, 9, 4417.

[5] S. Linic, P. Christopher, D. B. Ingram, Nature materials 2011, 10, 911.

[6] P. Christopher, H. Xin, A. Marimuthu, S. Linic, Nature Mat 2012, 11, 1044.

[7] S. Linic, U. Aslam, C. Boerigter, M. Morabito, Nature materials 2015, 14, 567.

[8] G. V. Hartland, Chemical reviews 2011, 111, 3858.

[9] J. B. Khurgin, U. Levy, ACS Photonics 2020, 7, 547.

[10] G. V. Hartland, L. V. Besteiro, P. Johns, A. O. Govorov, ACS Energy Lett. 2017, 2, 1641.

[11] S. Mukherjee, F. Libisch, N. Large, O. Neumann, L. V. Brown, J. Cheng, J. B. Lassiter, E. A. Carter, P. Nordlander, N. J. Halas, Nano letters 2013, 13, 240.

[12] a) E. Cortés, Advanced Optical Materials 2017, 5, 1700191; b) Y. Zhang, S. He, W. Guo, Y. Hu, J. Huang, J. R. Mulcahy, W. D. Wei, Chemical reviews 2018, 118, 2927.

[13] S. Mukherjee, L. Zhou, A. M. Goodman, N. Large, C. Ayala-Orozco, Y. Zhang, P. Nordlander, N. J. Halas, Journal of the American Chemical Society 2014, 136, 64.

[14] Z. Zheng, W. Xie, B. Huang, Y. Dai, Chemistry (Weinheim an der Bergstrasse, Germany) 2018, 24, 18322.

[15] S. Yu, P. K. Jain, Nature communications 2019, 10, 2022.

[16] F. Mohammadparast, A. P. Dadgar, R. T. A. Tirumala, S. Mohammad, C. O. Topal, A. K. Kalkan, M. Andiappan, J. Phys. Chem. C 2019, 123, 11539.

[17] R. M. Sarhan, W. Koopman, R. Schuetz, T. Schmid, F. Liebig, J. Koetz, M. Bargheer, Scientific reports 2019, 9, 3060.

[18] J.-J. Sun, H.-S. Su, H.-L. Yue, S.-C. Huang, T.-X. Huang, S. Hu, M. M. Sartin, J. Cheng, B. Ren, The journal of physical chemistry letters 2019, 10, 2306.

[19] F.-H. Cho, S.-C. Kuo, Y.-H. Lai, RSC Adv. 2017, 7, 10259.

[20] Q. Zhang, Y. Zhou, X. Fu, E. Villarreal, L. Sun, S. Zou, H. Wang, J. Phys. Chem. C 2019, 123, 26695. 
[21] A. A. Golubev, B. N. Khlebtsov, R. D. Rodriguez, Y. Chen, D. R. T. Zahn, J. Phys. Chem. C 2018, 122, 5657.

[22] E. L. Keller, R. R. Frontiera, ACS nano 2018, 12, 5848.

[23] J. L. Brooks, R. R. Frontiera, J. Phys. Chem. C 2016, 120, 20869.

[24] E. M. van Schrojenstein Lantman, O. L. J. Gijzeman, A. J. G. Mank, B. M.

Weckhuysen, ChemCatChem 2014, 6, 3342.

[25] H.-K. Choi, W.-H. Park, C.-G. Park, H.-H. Shin, K. S. Lee, Z. H. Kim, Journal of the American Chemical Society 2016, 138, 4673.

[26] Y. Dubi, Y. Sivan, Light, science \& applications 2019, 8, 89.

[27] a) L. Zhou, D. F. Swearer, C. Zhang, H. Robatjazi, H. Zhao, L. Henderson, L. Dong,

P. Christopher, E. A. Carter, P. Nordlander et al., Science (New York, N.Y.) 2018, 362, 69;

b) B. Y. Zheng, H. Zhao, A. Manjavacas, M. McClain, P. Nordlander, N. J. Halas, Nature communications 2015, 6, 7797.

[28] Y. Sivan, J. H. Baraban, Y. Dubi, OSA Continuum 2020, 3, 483.

[29] K. K. Kolasinski, Surface Science. Foundations of Catalysis and Nanoscience, John Wiley \& Sons, Hoboken, 2012.

[30] a) F. Liebig, R. M. Sarhan, M. Sander, W. Koopman, R. Schuetz, M. Bargheer, J. Koetz, ACS applied materials \& interfaces 2017, 9, 20247; b) R. M. Sarhan, W.

Koopman, J. Pudell, F. Stete, M. Rössle, M. Herzog, C. N. Z. Schmitt, F. Liebig, J. Koetz, M. Bargheer, J. Phys. Chem. C 2019, 123, 9352; c) R. Schürmann, K. Ebel, C. Nicolas,

A. R. Milosavljević, I. Bald, The journal of physical chemistry letters 2019, 10, 3153.

[31] a) Y.-F. Huang, H.-P. Zhu, G.-K. Liu, D.-Y. Wu, B. Ren, Z.-Q. Tian, J. Am. Chem. Soc. 2010, 132, 9244; b) D.-Y. Wu, X.-M. Liu, Y.-F. Huang, B. Ren, X. Xu, Z.-Q. Tian, J. Phys. Chem. C 2009, 113, 18212.

[32] Q. Cui, A. Yashchenok, L. Zhang, L. Li, A. Masic, G. Wienskol, H. Möhwald, M. Bargheer, ACS applied materials \& interfaces 2014, 6, 1999.

[33] Y. Ling, W. C. Xie, G. K. Liu, R. W. Yan, D. Y. Wu, J. Tang, Sci. Rep. 2016, 6, 31981.

[34] a) K. S. Shin, H. S. Lee, S. W. Joo, K. Kim, J. Phys. Chem. C 2007, 111, 15223; b) K. Kim, J.-Y. Choi, K. S. Shin, J. Phys. Chem. C 2014, 118, 11397.

[35] S. Sun, R. L. Birke, J. R. Lombardi, K. P. Leung, A. Z. Genack, J. Phys. Chem. 1988, $92,5965$.

[36] a) L. Cui, X. Ren, X. Yang, P. Wang, Y. Qu, W. Liang, M. Sun, J. Raman Spectrosc. 2016, 47, 877; b) V. Joseph, C. Engelbrekt, J. Zhang, U. Gernert, J. Ulstrup, J. Kneipp, Angewandte Chemie (International ed. in English) 2012, 51, 7592.

[37] X. Tang, W. Cai, L. Yang, J. Liu, Nanoscale 2014, 6, 8612.

[38] a) E. Cortés, W. Xie, J. Cambiasso, A. S. Jermyn, R. Sundararaman, P. Narang, S.

Schlücker, S. A. Maier, Nature communications 2017, 8, 14880; b) S. Pal, A. Dutta, M.

Paul, A. Chattopadhyay, J. Phys. Chem. C 2020, 124.

[39] Z. Zhang, T. Deckert-Gaudig, P. Singh, V. Deckert, Chemical communications

(Cambridge, England) 2015, 51, 3069.

[40] C. Boerigter, U. Aslam, S. Linic, ACS nano 2016, 10, 6108.

[41] Y.-Y. Cai, E. Sung, R. Zhang, L. J. Tauzin, J. G. Liu, B. Ostovar, Y. Zhang, W.-S.

Chang, P. Nordlander, S. Link, Nano Lett. 2019, 19, 1067.

[42] C. A. Lieber, A. Mahadevan-Jansen, Applied spectroscopy 2003, 57, 1363.

[43] P. W. Atkins, J. de Paula, Atkins' physical chemistry, Oxford University Press, Oxford, 2014. 